\title{
Strongly rational sets for normal-form games
}

Citation for published version (APA):

Grandjean, J. G., Mauleon, A., \& Vannetelbosch, V. (2009). Strongly rational sets for normal-form games. METEOR, Maastricht University School of Business and Economics. METEOR Research Memorandum No. 059 https://doi.org/10.26481/umamet.2009059

Document status and date:

Published: 01/01/2009

DOI:

10.26481/umamet.2009059

Document Version:

Publisher's PDF, also known as Version of record

\section{Please check the document version of this publication:}

- A submitted manuscript is the version of the article upon submission and before peer-review. There can be important differences between the submitted version and the official published version of record.

People interested in the research are advised to contact the author for the final version of the publication, or visit the DOI to the publisher's website.

- The final author version and the galley proof are versions of the publication after peer review.

- The final published version features the final layout of the paper including the volume, issue and page numbers.

Link to publication

\footnotetext{
General rights rights.

- You may freely distribute the URL identifying the publication in the public portal. please follow below link for the End User Agreement:

www.umlib.nl/taverne-license

Take down policy

If you believe that this document breaches copyright please contact us at:

repository@maastrichtuniversity.nl

providing details and we will investigate your claim.
}

Copyright and moral rights for the publications made accessible in the public portal are retained by the authors and/or other copyright owners and it is a condition of accessing publications that users recognise and abide by the legal requirements associated with these

- Users may download and print one copy of any publication from the public portal for the purpose of private study or research.

- You may not further distribute the material or use it for any profit-making activity or commercial gain

If the publication is distributed under the terms of Article $25 \mathrm{fa}$ of the Dutch Copyright Act, indicated by the "Taverne" license above, 


\section{Maastricht University}

Gilles Grandjean, Ana Mauleon, Vincent Vannetelbosch

Strongly Rational Sets for Normal-Form Games

$\mathrm{RM} / 09 / 059$

\section{METEOR}

Maastricht University School of Business and Economics

Maastricht Research School of Economics

of Technology and Organization

P.O. Box 616

NL - 6200 MD Maastricht

The Netherlands 


\title{
Strongly Rational Sets for Normal-Form Games ${ }^{\dagger}$
}

\author{
Gilles Grandjean $^{\mathrm{a}}$, Ana Mauleon ${ }^{\mathrm{b}, \mathrm{c}}$, Vincent Vannetelbosch ${ }^{\mathrm{c}}$ \\ ${ }^{\mathrm{a}}$ CORE, Université catholique de Louvain, 34 voie du Roman Pays, B-1348 \\ Louvain-la-Neuve, Belgium. \\ ${ }^{\mathrm{b}}$ FNRS and CEREC, Facultés universitaires Saint-Louis, Boulevard du Jardin \\ Botanique 43, B-1000 Brussels, Belgium. \\ ${ }^{\mathrm{c}}$ FNRS and CORE, Université catholique de Louvain, 34 voie du Roman Pays, \\ B-1348 Louvain-la-Neuve, Belgium.
}

October 27, 2009

\begin{abstract}
Curb sets [Basu and Weibull, Econ. Letters 36 (1991), 141-146] are product sets of pure strategies containing all individual best-responses against beliefs restricted to the recommendations to the remaining players. The concept of minimal curb sets is a set-theoretic coarsening of the notion of strict Nash equilibrium. We introduce the concept of minimal strong curb sets which is a set-theoretic coarsening of the notion of strong Nash equilibrium. Strong curb sets are product sets of pure strategies such that each player's set of recommended strategies must contain all coalitional bestresponses of each coalition to whatever belief each coalition member may have that is consistent with the recommendations to the other players. Minimal strong curb sets are shown to exist and are compared with other well known solution concepts. We also provide a dynamic learning process leading the players to playing strategies from a minimal strong curb set.
\end{abstract}

JEL classification: $\mathrm{C} 72$

Keywords: Set-valued solution concept, Coalitional best-response, Strong curb set, Learning.

\footnotetext{
${ }^{\dagger}$ Corresponding author: Prof. Vincent Vannetelbosch. E-mail addresses: gilles.grandjean@uclouvain.be (Gilles Grandjean), mauleon@fusl.ac.be (Ana Mauleon), vincent.vannetelbosch@uclouvain.be (Vincent Vannetelbosch).
} 


\section{Introduction}

The notion of Nash equilibrium does not incorporate the possibility that groups of players might coordinate their actions to reach an outcome that is better for all of them. Aumann (1959) was first to introduce this consideration into the theory of noncooperative games by proposing the notion of strong Nash equilibrium. A strategy profile is a strong Nash equilibrium if it is immune not only to individual deviations, but also to coalitional deviations. Later on, Bernheim, Peleg and Whinston (1987) have proposed the notion of coalition-proof Nash equilibrium. A strategy profile is a coalition-proof Nash equilibrium if it is immune to coalitional deviations which are themselves immune to further deviations by subcoalitions. The main weakness of strong Nash equilibrium and coalition-proof Nash equilibrium is that existence is not guaranteed in a natural class of games, as opposed to the Nash equilibrium concept.

Basu and Weibull (1991) have proposed a set-theoretic coarsening of the notion of strict Nash equilibrium: minimal curb (closed under rational behavior) sets. ${ }^{1}$ This set-valued solution concept combines a standard rationality condition, stating that the set of recommended strategies of each player must contain all best responses to whatever belief he may have that is consistent with the recommendations to the other players, with players' aim at simplicity, which encourages them to maintain a set of strategies as small as possible.

In this paper we introduce the concept of minimal strong curb sets which is a set-theoretic coarsenings of the notion of (strict) strong Nash equilibrium. We require the sets to be immune not only against individual deviations, but also against group deviations. Strong curb sets are product sets of pure strategies such that each player's set of recommended strategies must contain all coalitional best-responses of each coalition to whatever belief each coalition member may have that is consistent with the recommendations to the other players. A strong curb set is minimal if it does not properly contain another strong curb set. Think of the set of recommendations to a player in a minimal strong curb set as a well-packed bag for a sports

\footnotetext{
${ }^{1}$ Many games of interest lack strict Nash equilibria. A strategy profile is a strict Nash equilibrium if each player's equilibrium strategy is better than all her other strategies, given the other players' strategies. In any non-strict Nash equilibrium, at least one player is indifferent between her equilibrium strategy and some other strategy, given the other players' strategies. Such indifference can make the Nash equilibrium evolutionary unstable. See Weibull (1995).
} 
weekend: you may want to be prepared for different kinds of sports since you may like playing tennis with player 2 or playing golf with playing 3 or playing bridge with players 2, 3 and 4 or going alone for a jog. Minimal strong curb sets are shown to exist in general and are compared with other well known solution concepts: strong Nash equilibrium, coalition-proof Nash equilibrium, and coalitional rationalizability.

Finally, we provide a dynamic motivation for the concept of minimal strong curb sets. Hurkens (1995) has proposed a dynamic learning process where players have bounded memory and play best-responses against beliefs, formed on the basis of strategies used in the recent past. This learning process leads the players to playing strategies from a minimal curb set. ${ }^{2}$ We propose a similar learning process except that now groups of players may play coalitional best-responses. A game is played at discrete point in time. For each role in the game there is a pool of players. At the beginning of each period one player is drawn from each pool to play the game in that period. These players are partitioned into coalitions to form a coalition structure. Each coalition structure has a positive probability to occur at each period. Players observe how the game has been played in the recent past, form their beliefs upon these observations, and select an action profile jointly with their coalition partners. We show that, if the memory is long enough, play settle down in a minimal strong curb set.

The paper is organized as follows. We recall notations and definitions in Section 2. We formally define the concept of minimal strong curb sets in Section 3. We compare minimal strong curb sets with strong Nash equilibria, coalition-proof Nash equilibria and coalitionally rationalizable strategy profiles in Section 4 . We provide a dynamic learning process leading the players to playing strategies from a minimal strong curb set in Section 5. We conclude in Section 6.

\section{Preliminaries}

Strict set inclusion is denoted by $\varsubsetneqq$ and weak set inclusion is denoted by $\subseteq$. A normal-form game is a tuple $G=\left\langle N,\left\{A_{i}\right\}_{i \in N},\left\{u_{i}\right\}_{i \in N}\right\rangle$, where $N=\{1,2, \ldots, n\}$ is a finite set of players, each player $i \in N$ has a nonempty, finite set of pure strategies (or actions) $A_{i}$ and a von Neumann-Morgenstern utility function $u_{i}: A \rightarrow \mathbb{R}$, where $A=\times_{j \in N} A_{j}$. The set of all games is denoted by $\Gamma$. For every $X \subseteq A$, let $X_{-i}$

\footnotetext{
${ }^{2}$ See also Young (1998).
} 
$=\times_{j \in N \backslash\{i\}} X_{j}, \forall i \in N$. The subgame obtained from $G$ by restricting the action set of each player $i \in N$ to a subset $X_{i} \subseteq A_{i}$ is denoted - with a minor abuse of notation from restricting the domain of the utility functions $u_{i}$ to $\times_{j \in N} X_{j}$ - by $G_{X}=\left\langle N,\left\{X_{i}\right\}_{i \in N},\left\{u_{i}\right\}_{i \in N}\right\rangle$. The set of mixed strategies of player $i \in N$ with support in $X_{i} \subseteq A_{i}$ is denoted by $\Delta\left(X_{i}\right)$. Payoffs are extended to mixed strategies in the usual way. Beliefs are profiles of mixed strategies: correlation is not allowed. The profile of strategies where player $i \in N$ plays $a_{i} \in A_{i}$ and her opponents play according to the mixed strategy profile $\alpha_{-i}=\left(\alpha_{j}\right)_{j \in N \backslash\{i\}} \in \times_{j \in N \backslash\{i\}} \Delta\left(A_{j}\right)$ is denoted $\left(a_{i}, \alpha_{-i}\right)$. For $i \in N$ and $\alpha_{-i} \in \times_{j \in N \backslash\{i\}} \Delta\left(A_{j}\right)$,

$$
\operatorname{BR}^{i}\left(\alpha_{-i}\right)=\left\{a_{i} \in A_{i} \mid u_{i}\left(a_{i}, \alpha_{-i}\right) \geq u_{i}\left(a_{i}^{\prime}, \alpha_{-i}\right) \text { for each } a_{i}^{\prime} \in A_{i}\right\}
$$

is the set of pure best responses of player $i$ against her belief $\alpha_{-i}$.

Basu and Weibull (1991) have introduced the concept of strategy subset closed under rational behavior (curb), which is a set-theoretic coarsening of the notion of strict Nash equilibrium. Formally, curb sets are defined as follows.

Definition 1. A curb set is a product set $X=\times_{i \in N} X_{i}$ where

(a) for each $i \in N, X_{i} \subseteq A_{i}$ is a nonempty set of pure strategies;

(b) for each $i \in N$ and each belief $\alpha_{-i}$ of player $i$ with support in $X_{-i}$, the set $X_{i}$ contains all best responses of player $i$ against his belief:

$$
\forall i \in N, \forall \alpha_{-i} \in \times_{j \in N \backslash\{i\}} \Delta\left(X_{j}\right), \mathrm{BR}^{i}\left(\alpha_{-i}\right) \subseteq X
$$

Curb sets are product sets of pure strategies such that each player's set of recommended strategies must contain all best-replies to whatever belief he may have that is consistent with the recommendations to the other players. Since the full strategy space is always curb, particular attention is devoted to minimal curb sets. A curb set $X$ is minimal if no curb set is a proper subset of $X$. Basu and Weibull (1991) have shown that every game $G$ possesses at least one minimal curb set. The set-valued solution concept that assigns to each game its collection of minimal curb sets is denoted by min-curb. Hence, min-curb $(G)=\{X \subseteq A \mid X$ is a minimal curb set of $G\}$. Similarly, $\operatorname{curb}(G)=\{X \subseteq A \mid X \text { is a curb set of } G\}^{3}$

\footnotetext{
${ }^{3}$ Voorneveld (2004) has proposed the notion of prep sets which are product sets of pure strategies such that each player's set of recommended strategies must contain at least one best-response to
} 
The notion of strong Nash equilibrium is due to Aumann (1959). A strong Nash equilibrium is a strategy profile such that no subset of players has a joint deviation that benefits all of them. Coalitions are nonempty subsets of players $(J$ such that $J \subseteq N$ and $J \neq \varnothing)$. For every $X \subseteq A$, let $X_{-J}=\times_{j \in N \backslash J} X_{j}, \forall J \subseteq N$. The profile of strategies where players belonging to coalition $J$ play according to the strategy profile $a_{J} \in \times_{i \in J} A_{i}$ and the remaining players play according to the mixed strategy profile $\alpha_{-J}=\left(\alpha_{j}\right)_{j \in N \backslash J} \in \times_{j \in N \backslash J} \Delta\left(A_{j}\right)$ is denoted $\left(a_{J}, \alpha_{-J}\right)$. For every $J \subseteq N, i \in J, X \subseteq A$ and $\alpha_{-i}=\left(\alpha_{j}\right)_{j \in N \backslash\{i\}} \in \times_{j \in N \backslash\{i\}} \Delta\left(X_{j}\right)$, we denote by $\alpha_{-i}^{-J}$ the marginal distribution of $\alpha_{-i}$ over $X_{-J}$. Formally, the notion of strong Nash equilibrium is defined as follows. The strategy profile $a^{*} \in \times_{i \in N} A_{i}$ is a strong Nash equilibrium if and only if, $\forall J \subseteq N, \forall a_{J} \in \times_{j \in J} A_{j}\left(a_{J} \neq a_{J}^{*}\right), \exists i \in J$ such that $u_{i}\left(a^{*}\right) \geq u_{i}\left(a_{J}, a_{-J}^{*}\right)$. A strong Nash equilibrium is strict if the last inequality holds strictly.

\section{Strong curb sets}

While the concept of curb sets is a set-theoretic coarsening of the notion of strict Nash equilibrium, we now introduce the concept of strong curb sets which is a settheoretic coarsening of the notion of strict strong Nash equilibrium. That is, we require the set to be immune not only against individual deviations (as for curb sets), but also against coalitional deviations. Let us generalize the concept of best response to coalitions of players.

Definition 2. For each vector of beliefs $\boldsymbol{\alpha}=\left(\alpha_{-i}\right)_{i \in N}$ with $\alpha_{-i} \in \times_{j \in N \backslash\{i\}} \Delta\left(A_{j}\right)$, the set of coalitional best-responses of coalition $J \subseteq N$ is

$$
\begin{array}{r}
\operatorname{CBR}^{J}(\boldsymbol{\alpha})=\left\{a_{J} \in \times_{i \in J} A_{i} \mid(\mathrm{i}) \forall i \in J, u_{i}\left(a_{i}, \alpha_{-i}\right) \leq u_{i}\left(a_{J}, \alpha_{-i}^{-J}\right), \forall a_{i} \in A_{i}\right. \text { and } \\
\text { (ii) } \left.\nexists a_{J}^{\prime} \in \times_{i \in J} A_{i} \text { such that } \forall i \in J, u_{i}\left(a_{J}, \alpha_{-i}^{-J}\right)<u_{i}\left(a_{J}^{\prime}, \alpha_{-i}^{-J}\right)\right\} .
\end{array}
$$

whatever belief she may have that is consistent with the recommendations to the other players. A formal definition is provided in the appendix. Every curb set is a prep set and every curb set contains a minimal prep set. But, minimal prep sets may contain a proper subset of the strategies contained in the minimal curb sets. Kalai and Samet (1984) have introduced the notion of persistent retracts which require the recommendations to each player to contain at least one best-response to beliefs in a small neighborhood of the beliefs restricted to the recommendations to the other players. Voorneveld (2005) has shown that, in generic games, persistent retracts, minimal prep sets and minimal curb sets coincide. 
Given a vector of beliefs $\boldsymbol{\alpha}$, a profile of strategies $a_{J}$ for coalition $J$ is a coalitional best-response if (i) each member $i \in J$ prefers to join coalition $J$ and playing $a_{J}$ rather than playing her individually best-response against her belief $\alpha_{-i}$, (ii) there is no other profile $a_{J}^{\prime} \neq a_{J}$ such that all members of $J$ strictly prefer $a_{J}^{\prime}$ to $a_{J}$. Conditions (i) and (ii) captures some rudimentary form of coalitional rationality. First, a sensible concept of coalitional rationality should prescribe coordination on strategy profiles so that all coalition members have incentives to join the group. Second, it should be conceivable that members of coalition $J$ will never coordinate their play on strategy profiles that are Pareto dominated. Of course, $\operatorname{CBR}^{\{i\}}(\boldsymbol{\alpha})$ coincides with $\operatorname{BR}^{i}\left(\alpha_{-i}\right) \forall i \in N$.

Example 1. Consider the normal-form games $G_{1}$ and $G_{2}$.

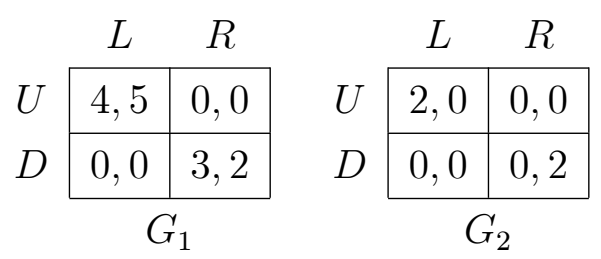

Take the normal-form game $G_{1}$ and let $J=\{1,2\}$. Condition (i) makes that $(U, R)$ and $(D, L)$ are never coalitional best-responses for $J$ whatever $\boldsymbol{\alpha}$. Condition (ii) makes that $(D, R)$ is not a coalitional best-response for $J$ whatever $\boldsymbol{\alpha}$. However, the strategy profile $(U, L)$ satisfies both conditions whatever $\boldsymbol{\alpha}$. Thus, $\operatorname{CBR}^{\{1,2\}}(\boldsymbol{\alpha})=$ $\{(U, L)\}$. Notice that the set of coalitional best-responses, $\operatorname{CBR}^{J}(\boldsymbol{\alpha})$, may be empty if $|J| \geq 2$. Take the normal-form game $G_{2}$ and consider the beliefs $\boldsymbol{\alpha}=\left(\alpha_{-1}, \alpha_{-2}\right)$ with $\alpha_{-1}(L)=1$ and $\alpha_{-2}(D)=1$. Then, $\operatorname{BR}^{1}\left(\alpha_{-1}\right)=\{U\}$ and $\operatorname{BR}^{2}\left(\alpha_{-2}\right)=\{R\}$ and the expected payoffs are $u_{1}\left(U, \alpha_{-1}\right)=2$ and $u_{2}\left(R, \alpha_{-2}\right)=2$. Thus, we have that $\operatorname{CBR}^{\{1,2\}}(\boldsymbol{\alpha})=\varnothing$.

A set $X$ is a strong curb set if the belief that only strategies in $X$ are played implies that players and coalitions have no incentives to use other strategies than those belonging to $X$. Formally, strong curb sets are defined as follows.

Definition 3. A strong curb set is a product set $X=\times_{i \in N} X_{i}$ where

(a) for each $i \in N, X_{i} \subseteq A_{i}$ is a nonempty set of pure strategies;

(b) for each $J \subseteq N$ and each vector of beliefs $\alpha=\left(\alpha_{-1}, \ldots, \alpha_{-N}\right)$ of the players with each belief $\alpha_{-i}$ having support in $X_{-i}$, the product set $X_{J}=\times_{j \in J} X_{j}$ 
contains all coalitional best-responses of coalition $J$ against the beliefs of its members:

$$
\begin{aligned}
& \forall J \subseteq N, \forall \boldsymbol{\alpha}=\left(\alpha_{-1}, \ldots, \alpha_{-n}\right) \text { with } \alpha_{-i} \in \times_{l \in N \backslash\{i\}} \Delta\left(X_{l}\right), i \in N, \\
& \mathrm{CBR}^{J}(\boldsymbol{\alpha}) \subseteq \times_{j \in J} X_{j} .
\end{aligned}
$$

Strong curb sets are product sets of pure strategies such that each player's set of recommended strategies must contain all coalitional best-responses of each coalition to whatever belief each coalition member may have that is consistent with the recommendations to the other players. ${ }^{4} \mathrm{~A}$ set $X \subseteq A$ is not a strong curb set if there exists a coalition having a deviation outside the set of recommended strategies such that each coalition member is at least as well off by deviating for at least one possible belief concerning the play of others in the set of recommended strategies. A deviation is blocked if we can find one player who is strictly better off by blocking the deviation. Notice that each coalition member is allowed to have a different belief concerning the play of others in the set of recommended strategies to assess the profitability of the deviation. In other words, the coalition members may disagree on where the deviation leads to. ${ }^{56}$

A strong curb set $X$ is minimal if no strong curb set is a proper subset of $X$. The set-valued solution concept that assigns to each game its collection of minimal strong curb sets is denoted by min-strong-curb. Hence, for a game $G$, min-strong-curb $(G)=\{X \subseteq A \mid X$ is a minimal strong curb set of $G\}$ and strong$\operatorname{curb}(G)=\{X \subseteq A \mid X$ is a strong curb set of $G\}$. Every normal-form game has a minimal strong curb set.

\footnotetext{
${ }^{4}$ We assume that players choose pure strategies. However, the notion of strong curb set can be easily extended to mixed strategies simply by accommodating the definition of CBR. Then, strong curb sets would still be product sets of pure strategies but such that each player's set of recommended strategies contains now the support all coalitional best-responses of each coalition to whatever belief each coalition member may have that is consistent with the recommendations to the other players.

${ }^{5}$ We are implicitly assuming that players do not update their beliefs by trying to understand why some coalitional action is a best-response for the other players of the coalition.

${ }^{6}$ Similarly to strong curb sets, we can define the notion of strong prep sets. Strong prep sets are product sets of pure strategies such that each player's set of recommended strategies must contain at least one coalitional best-response of each coalition to whatever belief each coalition member may have that is consistent with the recommendations to the other players. We provide a formal definition of strong prep sets in the appendix.
} 
Proposition 1. Every normal-form game $G$ has a minimal strong curb set.

Establishing existence of minimal strong curb sets in finite games is simple. The entire pure-strategy space $A$ is a strong curb set. Hence the collection of strong curb sets is nonempty, finite (since $A$ is finite) and partially ordered by set inclusion. Consequently, a minimal strong curb set exists. In the appendix we show that the existence result holds for every game $G \in \mathcal{G}$, where $\mathcal{G}$ is the class of normal-form games $G=\left\langle N,\left\{A_{i}\right\}_{i \in N},\left\{u_{i}\right\}_{i \in N}\right\rangle$ where for each player $i \in N=\{1,2, \ldots, n\}, A_{i}$ is a compact subset of a metric space and $u_{i}: A \rightarrow \mathbb{R}$ is a continuous von NeumannMorgenstern utility function.

If $X$ is a minimal strong curb set of $G=\left\langle N,\left\{A_{i}\right\}_{i \in N},\left\{u_{i}\right\}_{i \in N}\right\rangle$, then it is a minimal strong curb set of the subgame $G_{X}=\left\langle N,\left\{X_{i}\right\}_{i \in N},\left\{u_{i}\right\}_{i \in N}\right\rangle$. The intuition behind the proof of this result is the following. In the game $G$, for every possible belief profile with support in $X$, there is no profitable deviation outside $X$ (since $X \in \min$-strong-curb $(G))$. Then, there is no deviation from some subset $Y \subseteq X$ outside $X$ for beliefs with support in $Y$. Since $Y \notin \min$-strong-curb $(G)$ (as it would contradict that $X \in$ min-strong-curb $(G)$ ), there should exist a deviation from $Y$ to $X \backslash Y$. Then, $Y \notin$ min-strong-curb $\left(G_{X}\right)$.

Proposition 2. If $X \in \min$-strong-curb $(G)$ then $X \in \min$-strong-curb $\left(G_{X}\right)$.

Proof. Let $X \in$ min-strong-curb $(G) . X$ is a trivial strong curb set of the subgame $G_{X}: X \in \operatorname{strong-curb}\left(G_{X}\right)$. We will show that there is no $Y \varsubsetneqq X$ such that $Y \in$ strong-curb $\left(G_{X}\right)$. Suppose, on the contrary, that there exists $Y \varsubsetneqq X$ such that $Y \in$ strong-curb $\left(G_{X}\right)$. Since $Y$ is not a minimal strong curb set of $G$, there exists a vector of beliefs concentrated on $Y$ and a coalition $J \subseteq N$ such that each member of the coalition prefers to play a strategy profile outside the set $Y$ rather than playing a best-response in $Y$ to his belief. Formally, since $Y \notin$ min-strong$\operatorname{curb}(G)$, there exists $J \subseteq N, a_{J} \in \times_{j \in J} A_{j} \backslash Y_{j}$ and $\boldsymbol{\alpha}=\left(\alpha_{-1}, \ldots, \alpha_{-N}\right)$ with $\alpha_{-i} \in$ $\times_{j \in N \backslash\{i\}} \Delta\left(Y_{j}\right), i \in N$, such that $u_{j}\left(a_{J}, \alpha_{-j}^{-J}\right) \geq u_{j}\left(a_{j}, \alpha_{-j}\right)$ for all $j \in J$, for all $a_{j} \in Y_{j}$. Since $Y \in$ strong-curb $\left(G_{X}\right)$, the aforementioned deviation of coalition $J$ does not belong to $\times_{j \in J} X_{j} \backslash Y_{j}$, we have $a_{J} \in \times_{j \in J} A_{j} \backslash X_{j}$. Since $X \in \operatorname{strong-curb}(G)$ and $\alpha_{-i} \in \times_{j \in N \backslash\{i\}} \Delta\left(X_{j}\right) \forall i \in N\left(\right.$ since $\times_{j \in N \backslash\{i\}} \Delta\left(Y_{j}\right) \varsubsetneqq \times_{j \in N \backslash\{i\}} \Delta\left(X_{j}\right)$ ), at least one member $j^{*} \in J$ prefers to play a best-response in $X$ against the belief $\alpha_{-j^{*}}$ than playing according to $a_{J}$. Thus, we have $u_{j^{*}}\left(b_{j^{*}}, \alpha_{-j^{*}}\right)>u_{j^{*}}\left(a_{J}, \alpha_{-j^{*}}^{-J}\right)$ for some $b_{j^{*}} \in X_{j}$. Since $u_{j^{*}}\left(a_{J}, \alpha_{-j^{*}}^{-J}\right) \geq u_{j^{*}}\left(a_{j^{*}}, \alpha_{-j^{*}}\right)$ for all $a_{j^{*}} \in Y_{j^{*}}(Y \notin \operatorname{strong-curb}(G))$, 
we have $u_{j^{*}}\left(b_{j^{*}}, \alpha_{-j^{*}}\right)>u_{j^{*}}\left(a_{j^{*}}, \alpha_{-j^{*}}\right)$ for some $b_{j^{*}} \in X_{j^{*}}$, for all $a_{j^{*}} \in Y_{j^{*}}$. This contradicts the fact that $Y \in \operatorname{strong-curb}\left(G_{X}\right)$ since we have identified a belief $\boldsymbol{\alpha}$ which is such that $\mathrm{BR}^{j^{*}}\left(\alpha_{-j^{*}}\right) \nsubseteq Y$.

\section{Relationships with other solution concepts}

In this section we relate the concept of minimal strong curb set to the concepts of strong Nash equilibrium, coalition-proof Nash equilibrium and coalitional rationalizability. The product set of actions chosen in every strict strong Nash equilibrium is a minimal strong curb set. Conversely, for every minimal strong curb set composed of one action per player, the strategy profile in which each player selects this action is a strict strong Nash equilibrium. The main weakness of the strong Nash equilibrium concept is that it fails to exist in a natural class of games. However, the existence of minimal strong curb sets is guaranteed in general. The question we now address is whether minimal strong curb sets allow us to make reasonable predictions in games in which a strong Nash equilibrium does not exist. We provide below a game in which a strong Nash equilibrium does not exist but the unique minimal strong curb set is a proper subset of the full strategy space.

Example 2. Consider the normal-form game $G_{3}$.

\begin{tabular}{c|c|c|c|}
\multicolumn{1}{c}{} & \multicolumn{1}{c}{$L$} & \multicolumn{1}{c}{$C$} & \multicolumn{1}{c}{$R$} \\
\cline { 2 - 4 }$U$ & 4,4 & 0,5 & 0,0 \\
\cline { 2 - 4 }$M$ & 0,3 & 2,2 & 0,0 \\
\cline { 2 - 4 }$D$ & 0,0 & 0,0 & $a, 1$ \\
\cline { 2 - 4 } & & &
\end{tabular}

For $a<4$ the game $G_{3}$ has no strong Nash equilibrium while the minimal strong curb set is unique: min-strong-curb $\left(G_{3}\right)=\{\{U, M\} \times\{L, C\}\}$. Indeed, when each player believes that the other player plays in the set, each player's individual bestresponses lie in the set. In addition, any coalitional deviations outside the set is blocked by player 2 .

The collection of minimal strong curb sets may be composed of more elements than the product set of actions chosen in every strong Nash equilibria even when strong Nash equilibria exist. Consider again the game $G_{3}$ for $a>4$. The strategy profile $(D, R)$ is the unique strong Nash equilibrium of the game. The set composed of those actions is thus a minimal strong curb set. But, $\{U, M\} \times\{L, C\}$ is another 
minimal strong curb set. As a consequence, the unique strong Nash equilibrium may not be the only reasonable prediction in this game.

We now establish that if $X \subseteq A$ is a strong curb set and $a \in \times_{i \in N} X_{i}$ is a strict strong Nash equilibrium of the subgame restricted to $X$, then $a$ is a strict strong Nash equilibrium of the original game.

Proposition 3. For every game $G=\left\langle N,\left(A_{i}\right)_{i \in N},\left(u_{i}\right)_{i \in N}\right\rangle$, if $X \subseteq A$ is a strong curb set of $G$ and $a \in \times_{i \in N} X_{i}$ is a strict strong Nash equilibrium of the subgame $G_{X}=\left\langle N,\left(X_{i}\right)_{i \in N},\left(u_{i}\right)_{i \in N}\right\rangle$, then a is a strict strong Nash equilibrium of the original game $G$.

Proof. Consider a game $G=\left\langle N,\left(A_{i}\right)_{i \in N},\left(u_{i}\right)_{i \in N}\right\rangle$. By contradiction, suppose $X$ $\subseteq A$ is a strong curb set of $G, a \in \times_{i \in N} X_{i}$ is a strict strong Nash equilibrium of the subgame $G_{X}=\left\langle N,\left(X_{i}\right)_{i \in N},\left(u_{i}\right)_{i \in N}\right\rangle$ but $a$ is not a strict strong Nash equilibrium of the original game $G$. Since $a$ is not a strict strong Nash equilibrium of the original game $G$, there exists a coalition $J \subseteq N$ and a strategy profile $a_{J}^{\prime} \in \times_{j \in J} A_{j}$ which satisfies $u_{i}\left(a_{J}^{\prime}, a_{-J}\right) \geq u_{i}(a) \forall i \in J$. Since $X$ is a strong curb set of the original game, $a_{J}^{\prime} \in \times_{j \in J} X_{j}\left(a_{J}^{\prime} \notin \times_{j \in J}\left(A_{j} \backslash X_{j}\right)\right)$. It contradicts the fact that $a$ is a strict strong Nash equilibrium of the subgame $G_{X}=\left\langle N,\left(X_{i}\right)_{i \in N},\left(u_{i}\right)_{i \in N}\right\rangle$.

When a coalition-proof Nash equilibrium exists, its support is not necessarily contained in a minimal strong curb set, as the following example shows.

Example 3 (Ambrus, QJE 2006). Consider the normal-form game $G_{4}$.

\begin{tabular}{|c|c|c|c|}
\hline & $L$ & $C$ & $R$ \\
\hline$U$ & $2,1,0$ & $0,0,0$ & $-9,-9,-9$ \\
\hline M & $2,0,1$ & $1,0,2$ & $-9,-9,-9$ \\
\hline$D$ & $-9,-9,-9$ & $-9,-9,-9$ & $-9,-9,-9$ \\
\hline
\end{tabular}

\begin{tabular}{c|c|c|c|}
\multicolumn{1}{c}{} & \multicolumn{1}{c}{$L$} & \multicolumn{1}{c}{$C$} \\
\cline { 2 - 4 }$U$ & $1,2,0$ & $0,2,1$ & $-9,-9,-9$ \\
\cline { 2 - 4 }$M$ & $0,0,0$ & $0,1,2$ & $-9,-9,-9$ \\
\cline { 2 - 4 }$D$ & $-9,-9,-9$ & $-9,-9,-9$ & $-9,-9,-9$ \\
\cline { 2 - 4 } & \multicolumn{3}{|c}{$c$}
\end{tabular}

\begin{tabular}{c|c|c|c|}
\multicolumn{2}{c}{$L$} & \multicolumn{1}{c}{$C$} & $R$ \\
\cline { 2 - 4 }$U$ & $-9,-9,-9$ & $-9,-9,-9$ & $-9,-9,-9$ \\
\cline { 2 - 4 }$M$ & $-9,-9,-9$ & $-9,-9,-9$ & $-9,-9,-9$ \\
\cline { 2 - 4 }$D$ & $-9,-9,-9$ & $-9,-9,-9$ & $-8,-8,-8$ \\
\cline { 2 - 4 } & \multicolumn{3}{|c}{$r$}
\end{tabular}

The unique coalition-proof Nash equilibrium of $G_{4}$ is $(D, R, r)$, while the unique minimal strong curb set is min-strong-curb $\left(G_{3}\right)=\{\{U, M\} \times\{L, C\} \times\{l, c\}\}$. The 
predictions obtained under the minimal strong curb set seem more reasonable than the one given by the coalition-proof Nash equilibrium.

Outside the equilibrium framework Bernheim (1984) and Pearce (1984) have proposed the concept of rationalizability which consists of an iterative procedure that eliminates at each round strategies that are never best-response. Strategies that survive this iterative procedure are said to be rationalizable. Basu and Weibull (1991) have shown that every strategy contained in a minimal curb set is rationalizable. ${ }^{7}$ However, contrary to curb sets, strong curb sets may include strategies that are strictly dominated or even not rationalizable. ${ }^{8}$

Example 4. Consider the prisoners dilemma $G_{5}$.

$$
\begin{array}{l|l|l|}
\multicolumn{1}{c}{} & \multicolumn{1}{l}{L} & \multicolumn{1}{l}{R} \\
\cline { 2 - 3 } U & 2,2 & 0,3 \\
\cline { 2 - 3 } D & 3,0 & 1,1 \\
\cline { 2 - 3 } & &
\end{array}
$$

We have that the action $\mathrm{U}(\mathrm{L})$ is strictly dominated for player 1 (2) but belongs to the unique minimal strong curb set of $G_{5}$. Indeed, min-strong-curb $\left(G_{5}\right)=$ $\{\{U, D\} \times\{L, R\}\}$.

Ambrus (2006) has proposed the concept of coalitional rationalizability using an iterative procedure. ${ }^{9}$ The construction is similar to the original definition of rationalizability provided by Bernheim (1984) and Pearce (1984), except that not only never best-response strategies of individual players are deleted by the procedure, but also strategies of group of players. Strategies of group of players are deleted if it is in their mutual interest to restrict their play to the remaining set of strategies. The set of coalitionally rationalizable strategies is the set of strategies that survive the iterative procedure of restrictions. ${ }^{10}$

\footnotetext{
${ }^{7}$ See Bernheim (1984), Pearce (1984), Herings and Vannetelbosch (1999, 2000) for the definitions of rationalizability for normal-form games and of its refinements. The set of rationalizable strategies coincide with the maximal tight curb set where tight curb sets are curb sets which are identical with their own best responses.

${ }^{8}$ Hofbauer and Weibull (1996) have provided a class of evolutionary selection dynamics under which strictly dominated strategies do survive for some games.

${ }^{9}$ Ambrus (2009) has provided an alternative concept of best-response to coalitions of players and he has offered epistemic definitions of coalitional rationalizability in normal-form games.

${ }^{10}$ Another approach is Herings, Mauleon and Vannetelbosch (2004) who have introduced the notion of social rationalizability
} 
Coalitional rationalizability may have more cutting power than minimal strong curb sets, as the following example shows.

Example 5 (Ambrus, QJE 2006). Consider the normal-form game $G_{6}$.

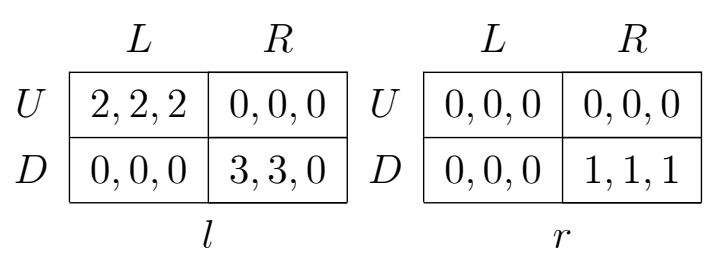

The game $G_{6}$ has a unique coalitionally rationalizable strategy profile which is $(D, R, r)$. Intuitively, player 1 and player 2 both recognize that they have a dominant strategy profile $(D, R)$. Anticipating this choice, player 3 selects $r$. On the other hand, $\{D\} \times\{R\} \times\{r\}$ is not a strong curb set since the deviation of the three players from $(D, R, r)$ to $(U, L, l)$ is Pareto improving. The unique strong curb set of $G_{6}$ is the full strategy space.

However, the converse may also be true. Minimal strong curb sets may have more cutting power than coalitional rationalizability.

Example 6 (Ambrus, QJE 2006). Consider the normal-form game $G_{7}$.

\begin{tabular}{c|c|c|c|}
\multicolumn{1}{c}{} & \multicolumn{1}{c}{$L$} & \multicolumn{1}{c}{$C$} & \multicolumn{1}{c}{$R$} \\
\cline { 2 - 4 }$U$ & $-2,1$ & $-1,0$ & $1,-2$ \\
\cline { 2 - 4 }$M$ & $0,-1$ & 0,0 & $0,-1$ \\
\cline { 2 - 4 }$D$ & $1,-2$ & $-1,0$ & $-2,1$ \\
\hline
\end{tabular}

In $G_{7}$ the strategy profile $(M, C)$ is a strict strong Nash equilibrium and min-strong$\operatorname{curb}\left(G_{7}\right)=\{\{M\} \times\{C\}\}$. But, any strategy profile is coalitionally rationalizable.

\section{$5 \quad$ Learning to play min-strong-curb strategies}

We now provide a class of dynamic learning processes in which groups of players may coordinate their actions. In line with Hurkens (1995), ${ }^{11}$ players observe actions played recently, form their beliefs upon these observations, and play best-responses to those beliefs. The new feature of the processes we propose is that players are

\footnotetext{
${ }^{11}$ See also Fudenberg and Levine (1998) or Young (1998). Kets and Voorneveld (2008) have provided an alternative dynamic learning process in which players display a bias towards recent choices and choose best-responses to beliefs supported by observed play in the recent past. The limit behavior of this learning process is shown to eventually settle down in minimal prep sets.
} 
allowed to play coalitional best-reponses. That is, players are allowed to select a joint action if by doing so, the expected payoff of each member of the group is increased with respect to the payoff she would have obtained by playing individually. We will show that the learning processes we propose lead the players to play only strategies from a minimal strong curb set, and thus provide a dynamic motivation for the concept of minimal strong curb set.

A game $G=\left\langle N,\left\{A_{i}\right\}_{i \in N},\left\{u_{i}\right\}_{i \in N}\right\rangle$ is played once every period. In each period, one player is drawn at random from each of $n$ disjoint classes $C_{1}, C_{2}, \ldots, C_{n}$, to play the game $G$ in that period. These players are partitioned into coalitions to form a coalition structure. A coalition structure $\mathbf{J}=\left(J_{1}, J_{2}, \ldots, J_{M}\right)$ is a partition of the player set $N=\{1,2, \ldots, n\}$ such that $J_{k} \cap J_{l}=\varnothing$ for $k \neq l$ and $\cup_{k=1}^{M} J_{k}=N$. Let $\mathbb{J}$ be the finite set of coalition structures. Each coalition structure $\mathbf{J} \in \mathbb{J}$ has a positive probability to occur at each period. Players have information about how the game has been played in the last $K$ periods. We define the state space $H=A^{K}$ to consist of all histories $h=\left(a^{-K}, \ldots, a^{-1}\right)$ of length $K$. In a given period $t$, for a particular history $h^{t}=\left(a^{t-K}, \ldots, a^{t-1}\right), a^{t-k}$ is the action profile chosen by the $n$ players in period $t-k$ for $k \in\{1, \ldots, K\}$. Since the choices of the players are time-independent, the learning process can be described by a stationary Markov chain on the state space $H=A^{K}$. Call $\widehat{h} \in H$ a successor of $h \in H$ if $\widehat{h}$ is obtained from $h$ by deleting the leftmost element and by adding some element $a \in A$ to the right. Let $r(\widehat{h})$ denote the rightmost element of $\widehat{h} \in H$. For $h=\left(a^{-K}, \ldots, a^{-1}\right) \in H$, let $\pi_{i}(h, k)=\left\{a_{i}^{-k}, \ldots, a_{i}^{-1}\right\}$ denote the set of strategies played by player $i$ in the $k$ last periods, for $k \leq K$. Let $P: H \times H \rightarrow[0,1]$ be a transition matrix, where $P(h, \widehat{h})$ is the probability of moving from state $h \in H$ to state $\widehat{h} \in H$ in one period and $\Sigma_{\widehat{h} \in H} P(h, \widehat{h})=1$ for all $h \in H$. A learning process is described by a transition matrix $P \in \mathcal{P}$, where $\mathcal{P}$ is defined as follows.

Definition 4. Let $\mathcal{P}$ be the set of transition matrices $P$ that satisfy for all histories $h$, $\widehat{h} \in H, P(h, \widehat{h})>0$ if and only if (i) $\widehat{h}$ is a successor of $h$, (ii) there exists some $\mathbf{J} \in \mathbb{J}$ and $\boldsymbol{\alpha}=\left(\alpha_{-1}, \ldots, \alpha_{-n}\right)$ with $\alpha_{-i} \in \times_{j \in N \backslash\{i\}} \Delta\left(\pi_{j}(h, K)\right)$ such that $r(\widehat{h})=\left(a_{J}\right)_{J \in \mathbf{J}}$ with $a_{J} \in \mathrm{CBR}^{J}(\boldsymbol{\alpha})$ if $\mathrm{CBR}^{J}(\boldsymbol{\alpha}) \neq \varnothing$ and $a_{J} \in \times_{i \in J} B R^{i}\left(\alpha_{-i}\right)$ otherwise.

At each period every player chooses an action. This action can be chosen individually or in group, and is chosen after having observed the recent past play. When a group of players coordinate their actions, they choose a Pareto undominated action 
profile such that each member of the group benefits from playing jointly. In state $h$, if coalition $J \subseteq N$ has a coalitional best-response $a_{J} \in \mathrm{CBR}^{J}(\boldsymbol{\alpha})$ given a profile of beliefs with support in the set of strategies played in the recent past, then the process moves with positive probability from state $h$ to state $\widehat{h}$ in which each member of coalition $J$ plays according to $a_{J}$. To determine the outcome of such learning processes, what matters is to identify, for each state $h$, the set of states that can be reached from $h$ in one period with positive probability and those that cannot be reached. Since the exact probability does not matter, we do not have to specify a particular process of belief formation nor a protocol of coalition formation. We only require that every such belief with support in the set of actions played recently and every partition of the players occur with positive probability.

For each $k \in N, P^{k}: H \times H \rightarrow[0,1]$ denotes the $k$-step transition probabilities of the Markov process with transition matrix $P \in \mathcal{P}: P^{1}=P$ and $P^{k}=P \circ P^{k-1}$ for $k>1$. We will write $h \rightsquigarrow \widehat{h}$ if there exists $k \in \mathbb{N}$ satisfying $P^{k}(h, \widehat{h})>0$. Now $\rightsquigarrow$ defines a weak order on $H$. We can define an equivalence relation on $H$ : $h \sim \widehat{h} \Leftrightarrow h \rightsquigarrow \widehat{h}$ and $\widehat{h} \rightsquigarrow h$. Let $[h]$ denote the equivalence class that contains $h$ and let $Q=\{[h] \mid h \in H\}$ denote the set of equivalence classes. A partial order $\preceq$ on $Q$ is given by: $[h] \preceq[\widehat{h}] \Leftrightarrow \widehat{h} \rightsquigarrow h$. The minimal elements with respect to the order $\preceq$ are called ergodic sets. The other elements are called transient sets. If the process leaves a transient set it can never return to that set. If the process is in an ergodic set it can never leave that set. The elements belonging to ergodic and transient sets are called ergodic and transient states, respectively. In any finite Markov chain, no matter where the process starts, the probability that the process is in an ergodic state after $k$ steps tends to 1 as $k$ tends to infinity (see Kemeny and Snell, 1976). Proposition 4 states that if memory is long enough ( $K$ high enough), the probability that the players are playing a minimal strong curb strategy profile after $k$ steps of the learning process tends to 1 as $k$ tends to infinity. To prove this result it is sufficient to show that each ergodic set $Z$ of every Markov chain with transition matrix $P \in \mathcal{P}$ satisfies $Z \subseteq X^{K}$ for some $X \in \min$-strong-curb $(G)$.

Proposition 4. There exists $\underline{K} \in \mathbb{N}$ such that for all finite $K \geq \underline{K}$ and every Markov chain with transition matrix $P \in \mathcal{P}$, if $Z \subseteq H$ is an ergodic set then $Z \subseteq X^{K}$ for some minimal strong curb set $X$.

Let $L=\sum_{i=1}^{n}\left|A_{i}\right|-(n-1)$. Let $M=\max \left(\left|A_{1}\right|, \ldots,\left|A_{n}\right|, \Sigma_{i=1}^{n}\left|A_{i}\right|-n\right)$. Take 
$\underline{K}=L+M$ and let $K \geq \underline{K}$ be finite. Let $P \in \mathcal{P}$. To prove Proposition 4 we will show that (i) from any history $h^{1} \in H$, the process moves with positive probability in $L-1$ steps to a state $h^{L} \in H$ such that $\times_{i \in N} \pi_{i}\left(h^{L}, L\right)$ is a strong curb set, (ii) from state $h^{L}$, the process moves with positive probability in $M$ steps to a state $h^{L+M} \in H$ such that $\times_{i \in N} \pi_{i}\left(h^{L+M}, M\right)$ is a minimal strong curb set, and (iii) steps (i) and (ii) imply that if $Z \subseteq H$ is an ergodic set then $Z \subseteq X^{K}$ for some minimal strong curb set $X$. The following lemma will be useful to prove Proposition 4 .

Lemma 1. Let $h^{t}=\left(x^{K-t}, \ldots, x^{1}, a^{1}, \ldots, a^{t}\right)$ be a particular history. (a) If the players draw their beliefs from $\times_{i \in N} \pi_{i}\left(h^{t}, t\right)$, the process moves with positive probability to an history $h^{t+1}$ such that $\times_{i \in N} \pi_{i}\left(h^{t}, t\right) \varsubsetneqq \times_{i \in N} \pi_{i}\left(h^{t+1}, t+1\right)$ if $\times_{i \in N} \pi_{i}\left(h^{t}, t\right)$ is not a strong curb set. (b) If the players draw their beliefs from $\times_{i \in N} \pi_{i}\left(h^{t}, t\right)$, the process moves with probability 1 to an history $h^{t+1}$ such that $\times_{i \in N} \pi_{i}\left(h^{t}, t\right)=\times_{i \in N} \pi_{i}\left(h^{t+1}, t+\right.$ 1) if $\times_{i \in N} \pi_{i}\left(h^{t}, t\right)$ is a strong curb set.

Proof. Let $h^{t}=\left(x^{K-t}, \ldots, x^{1}, a^{1}, \ldots, a^{t}\right)$ be a particular history. (a) Assume that $\times_{i \in N} \pi_{i}\left(h^{t}, t\right)$ is not a strong curb set. Then, there exists a partition $\mathbf{J} \in \mathbb{J}$, a profile of beliefs with support in the set of actions played in the last $t$ periods $\boldsymbol{\alpha}=\left(\alpha_{-1}, \ldots, \alpha_{-n}\right)$ with $\alpha_{-i} \in \times_{j \in N \backslash\{i\}} \Delta\left(\pi_{j}\left(h^{t}, t\right)\right)$, and a profile of actions $a^{t+1} \in A \backslash \times_{i \in N} \pi_{i}\left(h^{t}, t\right)$ where $a^{t+1}=\left(b_{J}\right)_{J \in \mathbf{J}}$ with $b_{J} \in \operatorname{CBR}^{J}(\boldsymbol{\alpha})$ if $\operatorname{CBR}^{J}(\boldsymbol{\alpha}) \neq \varnothing$ and $b_{J} \in \times_{i \in J} \operatorname{BR}^{i}\left(\alpha_{-i}\right)$ otherwise. Let $h^{t+1}=\left(x^{K-t+1}, \ldots, x^{1}, a^{1}, \ldots, a^{t+1}\right)$. Then $P\left(h^{t}, h^{t+1}\right)>0$ and $\times_{i \in N} \pi_{i}\left(h^{t}, t\right) \varsubsetneqq \times_{i \in N} \pi_{i}\left(h^{t+1}, t+1\right)$.

(b) Assume that $\times_{i \in N} \pi_{i}\left(h^{t}, t\right)$ is a strong curb set. Take any partition $\mathbf{J} \in \mathbb{J}$ and any profile of beliefs with support in the set of actions played in the last $t$ periods $\left(\boldsymbol{\alpha}=\left(\alpha_{-1}, \ldots, \alpha_{-n}\right)\right.$ with $\left.\alpha_{-i} \in \times_{j \in N \backslash\{i\}} \Delta\left(\pi_{j}\left(h^{t}, t\right)\right)\right)$, we have that every profile of actions $a^{t+1} \in A$ such that $a^{t+1}=\left(b_{J}\right)_{J \in \mathbf{J}}$ with $b_{J} \in \operatorname{CBR}^{J}(\boldsymbol{\alpha})$ if $\operatorname{CBR}^{J}(\boldsymbol{\alpha}) \neq \varnothing$ and $b_{J} \in \times_{i \in J} \mathrm{BR}^{i}\left(\alpha_{-i}\right)$ otherwise, belongs to the strong curb set $\times_{i \in N} \pi_{i}\left(h^{t}, t\right)$ by definition of $P$. Let $h^{t+1}=\left(x^{K-t+1}, \ldots, x^{1}, a^{1}, \ldots, a^{t+1}\right)$. Then $P\left(h^{t}, h^{t+1}\right)>0$ and $\times_{i \in N} \pi_{i}\left(h^{t}, t\right)=\times_{i \in N} \pi_{i}\left(h^{t+1}, t+1\right)$.

Proof of Proposition 4. (i) Let $a^{1}, \ldots, a^{T} \in A$ be such that $a^{t+1} \notin \times_{i \in N} \pi_{i}\left(h^{t}, t\right)$ for all $t=1, \ldots, T-1$. By definition of $L$, we have $T \leq L$ since $\times_{i \in N} \pi_{i}\left(h^{1}, 1\right)$ contains $n$ actions, $\times_{i \in N} \pi_{i}\left(h^{t+1}, t+1\right)$ contains at least one additional action than $\times_{i \in N} \pi_{i}\left(h^{t}, t\right)$ and the action space $A$, which is the largest strong curb set, contains $\sum_{i=1}^{n}\left|A_{i}\right|$ of them. Thus, there exists a $\tau \leq L$ such that, starting from $h^{1}$ and applying $\tau$ times part (a) of Lemma 1 , we have $h^{1} \rightsquigarrow h^{\tau}=\left(x^{K-\tau}, \ldots, x^{1}, a^{1}, \ldots, a^{\tau}\right)$ 
and $\times_{i \in N} \pi_{i}\left(h^{\tau}, \tau\right)$ is a strong curb set. From part (b) of Lemma 1, we have $h^{\tau} \rightsquigarrow$ $h^{L}=\left(x^{K-L}, \ldots, x^{1}, a^{1}, \ldots, a^{\tau}, \ldots, a^{L}\right)$ such that $\times_{i \in N} \pi_{i}\left(h^{\tau}, \tau\right)$ is a strong curb set and $\times_{i \in N} \pi_{i}\left(h^{L}, L\right)=\times_{i \in N} \pi_{i}\left(h^{\tau}, \tau\right)$.

(ii) Let $X \subseteq \times_{i \in N} \pi_{i}\left(h^{L}, L\right)$ be a minimal strong curb set. Since $K \geq L+M$ and since every strategy in a minimal strong curb set is an element of a coalitional best-response to some belief concentrated on the set, there exists a set $\left\{b^{1}, \ldots, b^{M}\right\}$ that spans $X$ and such that $h^{L} \rightsquigarrow h^{L+M}=\left(\ldots, a^{1}, \ldots, a^{L}, b^{1}, \ldots, b^{M}\right)$. That is, from $h^{L}$ there is a positive probability that each player $i \in N$ draws specific beliefs from $\times_{j \in N \backslash\{i\}} \Delta\left(\pi_{j}\left(h^{L}, L\right)\right)$ and is assigned to specific coalitions during $M$ periods in a row (from period $L+1$ to period $L+M$ ) such that each coalition (possibly a single player) chooses a coalitional best-response in each period and the process reaches $h^{L+M}=$ $\left(\ldots, a^{1}, \ldots, a^{L}, b^{1}, \ldots b^{M}\right) .{ }^{12}$ Once in $h^{L+M}$, each player draws with positive probability her beliefs from the minimal strong curb set $\times_{i \in N} \Delta\left(\pi_{i}\left(h^{L+M}, M\right)\right)$ during $K-M$ periods in a row. Then, the process reaches history $h^{L+K}=\left(b^{1}, \ldots, b^{M}, c^{1}, \ldots, c^{K-M}\right)$ such that $\times_{i \in N} \pi_{i}\left(h^{L+K}, K-M\right) \subseteq X$. By definition of $\mathcal{P}$, when the process reaches state $h^{L+K}$, each player draws her beliefs from $X$ with probability 1 and plays coalitional best-responses to her beliefs by selecting with probability one actions from $X$. So, $\times_{i \in N} \pi_{i}\left(h^{L+K+1}, K\right) \subseteq X$. Repeating the previous argument, we have that $\times_{i \in N} \pi_{i}\left(h^{L+K+k}, K\right) \subseteq X$ for all $k \in \mathbb{N}$ and for all $h^{L+K+k}$ such that $h^{L+K} \rightsquigarrow h^{L+K+k}$. Once in $h^{L+K}$, each player plays with probability one actions from the minimal strong curb set $X$ in all future periods. The set $X^{K}$ thus contains an ergodic set.

(iii) By contradiction, suppose there exists an ergodic set $Z$ such that $Z \nsubseteq X^{K}$ for any minimal strong curb set $X$. Thus $Z$ contains an ergodic state $h \in H$ such that $h \notin X^{K}$ for all minimal strong curb set $X$. Applying (i) and (ii), we have $h \rightsquigarrow h^{\prime}$ such that $h^{\prime} \in Y^{K}$ for some minimal strong curb set $Y$ and $h^{\prime}$ is an ergodic state of some ergodic set $W \subseteq Y^{K}$. Since $h \notin W$, we do not have $h^{\prime} \rightsquigarrow h$. This contradicts the fact that $h$ is an ergodic state and thus that $Z$ is an ergodic set.

Remark 1. Take any game $G=\left\langle N,\left\{A_{i}\right\}_{i \in N},\left\{u_{i}\right\}_{i \in N}\right\rangle$ such that $\left|A_{i}\right|>1$ and $\left|A_{j}\right|>$ 1 for $i, j \in N, i \neq j$. We have $M=\sum_{i=1}^{n}\left|A_{i}\right|-n$. Suppose we are in state $h^{L}$ and $X \subseteq \times_{i \in N} \pi_{i}\left(h^{L}, L\right)$ is a minimal strong curb set of $G$. Let $k=\max \left(\left|X_{1}\right|, \ldots,\left|X_{n}\right|\right)$ and let $l=\sum_{i=1}^{n}\left|X_{i}\right|-n$. (a) If $X=\times_{i \in N} X_{i}$ is such that every action $a_{i} \in X_{i}$ of each player $i \in N$ is an individual best-response to some belief in the set, the process can converge in exactly $k$ periods from $h^{L}$ to $h^{L+k}=\left(\ldots, a^{1}, \ldots, a^{L}, b^{1}, \ldots b^{k}\right)$

\footnotetext{
${ }^{12}$ See Remark 1 for an explanation of the value of M.
} 
with the property that $\left\{b^{1}, \ldots, b^{k}\right\}$ spans $X$. (b) If $X=\times_{i \in N} X_{i}$ is such that some action $a_{i} \in X_{i}$ of player $i$ only belongs to some coalitional best-response, the time of convergence of the process can be longer even more if the same player is involved in different coalitional moves. It can take at most $l$ periods to move from $h^{L}$ to $h^{L+l}=\left(\ldots, a^{1}, \ldots, a^{L}, b^{1}, \ldots b^{l}\right)$ with the property that $\left\{b^{1}, \ldots, b^{l}\right\}$ spans $X$. This is illustrated through the following example.

Example 7. Consider the normal form game $G_{8}$

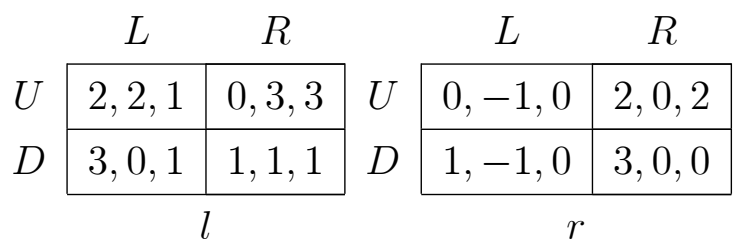

We have that min-strong-curb $\left(G_{8}\right)=\{\{U, D\} \times\{L, R\} \times\{l, r\}\}$ and $M=\sum_{i=1}^{n}\left|A_{i}\right|-$ $n=3$. Suppose the process is in state $h^{M}$ where $\times_{i \in N} \pi_{i}\left(h^{M}, M\right)=A$. Let $k$ be the smallest integer such that $h^{M} \rightsquigarrow h^{M+k}$ with the property that $\times_{i \in N} \pi_{i}\left(h^{M+k}, k\right)=A$. We have $k=3$ since player 2 selects her strategy $L$ only when coalition $\{1,2\}$ plays $(U, L)$. Player 3 selects her strategy $r$ only when coalition $\{1,3\}$ plays $(U, r)$. A third period is needed for player 1 to play $D$.

\section{Conclusion}

Basu and Weibull (1991) have introduced the notion of curb sets which are product sets of pure strategies containing all individual best-responses against beliefs restricted to the recommendations to the remaining players. The concept of minimal curb sets is a set-theoretic coarsening of the notion of strict Nash equilibrium. In this paper we have introduced the concept of minimal strong curb sets which is a set-theoretic coarsening of the notion of strong Nash equilibrium. Strong curb sets require sets to be immune not only against individual deviations, but also against group deviations. Strong curb sets are product sets of pure strategies such that each player's set of recommended strategies must contain all coalitional best-responses of each coalition to whatever belief each coalition member may have that is consistent with the recommendations to the other players. We have shown that minimal strong curb sets exist in general. We have also compared minimal strong curb sets with other well known solution concepts. Finally, we have provided a dynamic learning process leading the players to playing strategies from a minimal strong curb set only. 


\section{Acknowledgments}

We thank Olivier Tercieux and Jorgen Weibull for useful comments and suggestions. This paper has been presented at CORE, London School of Economics (EDP 2009), Maastricht University (CTN 2009), Northwestern University (GAMES 2008), SUNY at Stony Brook, University of Galway (PET 2009) and WEDI 2008 (Brussels). Ana Mauleon and Vincent Vannetelbosch are Research Associates of the National Fund for Scientific Research (FNRS), Belgium. Vincent Vannetelbosch is Associate Fellow of CEREC, Facultés Universitaires Saint-Louis. Financial support from Spanish Ministerio de Educación y Ciencia under the project SEJ2006-06309/ECON, support from the Belgian French Community's program Action de Recherches Concertée 05/10-331 (UCL), and support of a SSTC grant from the Belgian State - Belgian Science Policy under the IAP contract P6/09 are gratefully acknowledged.

\section{Appendix}

\section{A Existence of strong curb sets}

We now show that the existence of minimal strong curb sets holds in general. Let $\mathcal{G}$ be the class of normal-form games $G=\left\langle N,\left\{A_{i}\right\}_{i \in N},\left\{u_{i}\right\}_{i \in N}\right\rangle$ where for each player $i \in N=\{1,2, \ldots, n\}, A_{i}$ is a compact subset of a metric space and $u_{i}: A \rightarrow \mathbb{R}$ is a continuous von Neumann-Morgenstern utility function. Payoffs are extended to mixed strategies in the usual way. Let $\Delta\left(A_{i}\right)$ be the set of Borel probability measures over $A_{i}$. If $B_{i} \subseteq A_{i}$ is a Borel set, then $\Delta\left(B_{i}\right)$ denotes the set of Borel probability measures with support in $B_{i}: \Delta\left(B_{i}\right)=\left\{\alpha_{i} \in \Delta\left(A_{i}\right) \mid \alpha_{i}(B)=1\right\}$. If $G \in \mathcal{G}$, that is, payoff functions are continuous and strategy sets compact, then each set $\operatorname{BR}^{i}\left(\alpha_{-i}\right) \subseteq A_{i}$ is nonempty and compact.

Theorem A.1. Every game $G \in \mathcal{G}$ has a minimal strong curb set.

Proof. Let $Q=$ strong-curb $(G)$ denote the collection of all strong curb sets of $G$. $A$ is a strong curb set of $G$ since for every $J \subseteq N$ and $\boldsymbol{\alpha}=\left(\alpha_{-1}, \ldots, \alpha_{-N}\right)$ with $\alpha_{-i} \in \times_{l \in N \backslash\{i\}} \Delta\left(A_{l}\right), i \in N$, we have $\operatorname{CBR}^{J}(\alpha) \subseteq \times_{j \in J} A_{j}$. So $Q$ is nonempty and partially ordered via set inclusion. According to the Hausdorff Maximality Principle, $Q$ contains a maximal nested subset $R$. For each $i \in N$, let $X_{i}=\cap_{Y \in R} Y_{i}$ be the intersection of player $i$ 's strategies in the nested set $R$. The set $X_{i}$ is nonempty 
since the conditions of the Cantor intersection principle ${ }^{13}$ are satisfied, i.e. (i) the collection $\left\{Y_{i} \mid Y \in R\right\}$ is nested and thus satisfies the finite intersection property and (ii) each $Y_{i}$ is nonempty and compact for each strong curb set. It remains to prove that $X=\times_{i \in N} X_{i}$ is a minimal strong curb set. Take $\boldsymbol{\alpha}=\left(\alpha_{-1}, \ldots, \alpha_{-N}\right)$ with $\alpha_{-i} \in \times_{l \in N \backslash\{i\}} \Delta\left(X_{l}\right), i \in N$. We have that $\operatorname{CBR}^{J}(\boldsymbol{\alpha}) \cap \times_{j \in J}\left(A_{j} \backslash X_{j}\right)=\emptyset$ for $J \subseteq N$ since $\operatorname{CBR}^{J}(\boldsymbol{\alpha}) \cap \times_{j \in J}\left(A_{j} \backslash X_{j}\right)=\operatorname{CBR}^{J}(\boldsymbol{\alpha}) \cap\left(\cup_{Y \in R} \times_{j \in J}\left(A_{j} \backslash Y_{j}\right)\right)=$ $\cup_{Y \in R}\left(\mathrm{CBR}^{J}(\boldsymbol{\alpha}) \cap \times_{j \in J}\left(A_{j} \backslash Y_{j}\right)\right)$ and $\operatorname{CBR}^{J}(\boldsymbol{\alpha}) \cap \times_{j \in J}\left(A_{j} \backslash Y_{j}\right)=\emptyset$ for all $Y \in R(Y$ is a strong curb set). This establishes that $X$ is a strong curb set. The fact that it is minimal follows directly from the fact that $R$ is a maximal nested subset of $Q$.

\section{B Strong prep sets}

Voorneveld (2004) has proposed another set-valued solution concept, prep sets, which are formally defined as follows.

Definition B.1. A prep set is a product set $X=\times_{i \in N} X_{i}$ where (a) for each $i \in N$, $X_{i} \subseteq A_{i}$ is a nonempty set of pure strategies; (b) for each $i \in N$ and each belief $\alpha_{-i}$ of player $i$ with support in $X_{-i}$, the set $X_{i}$ contains at least one best response of player $i$ against his belief : $\forall i \in N, \forall \alpha_{-i} \in \times_{j \in N \backslash\{i\}} \Delta\left(X_{j}\right), B R^{i}\left(\alpha_{-i}\right) \cap X_{i} \neq \varnothing$.

A prep set $X$ is minimal if no prep set is a proper subset of $X$. Voorneveld (2004) has shown that every game $G$ possesses at least one minimal prep set. The set-valued solution concept that assigns to each game its collection of minimal prep sets is denoted by min-prep. ${ }^{14}$ Similarly to strong curb sets, we can define the notion of strong prep sets as follows.

Definition B.2. A strong prep set is a product set $X=\times_{i \in N} X_{i}$ where (a) for each $i \in N, X_{i} \subseteq A_{i}$ is a nonempty set of pure strategies; (b) for each $J \subseteq N$ and each vector of beliefs $\boldsymbol{\alpha}=\left(\alpha_{-1}, \ldots, \alpha_{-N}\right)$ of the players with each belief $\alpha_{-i}$ having support in $X_{-i}$, the product set $X_{J}=\times_{j \in J} X_{j}$ contains at least one coalitional best response

\footnotetext{
${ }^{13}$ In words, the Cantor intersection principle tells us that to show that the intersection of an infinite number of elements of a set $Z$ is nonempty and compact, we just need to show that the intersection is nonempty and compact for every subset of $Z$ composed of finite elements.

${ }^{14}$ Voorneveld, Kets and Norde (2005) have provided axiomatizations of minimal prep sets and minimal curb sets.
} 
of coalition $J$ against the beliefs of its members: $\forall J \subseteq N, \forall \boldsymbol{\alpha}=\left(\alpha_{-1}, \ldots, \alpha_{-n}\right)$ with $\alpha_{-i} \in \times_{l \in N \backslash\{i\}} \Delta\left(X_{l}\right), i \in N, C B R^{J}(\boldsymbol{\alpha}) \cap \times_{j \in J} X_{j} \neq \varnothing$.

A strong prep set $X$ is minimal if no strong prep set is a proper subset of $X$. Every strong curb set is a strong prep set, so if a strong curb set is contained in a minimal strong prep set, the two sets are necessarily equal. Similarly to Proposition 2 we have that, if $X$ is a minimal strong prep set of $G$, then it is a minimal strong prep set of the subgame $G_{X}$.

\section{References}

[1] Ambrus, A., "Coalitional rationalizability," Quarterly Journal of Economics 121, 903-929 (2006).

[2] Ambrus, A., "Theories of coalitional rationality," Journal of Economic Theory 144, 676-695 (2009).

[3] Aumann, R.J., "Acceptable points in general cooperative n-person games," in Contributions to the theory of games IV, Princeton Unviversity Press, pp.287324 (1959).

[4] Basu, K. and J.W. Weibull, "Strategy subsets closed under rational behavior," Economic Letters 36, 141-146 (1991).

[5] Bernheim, B.D., "Rationalizable Strategic Behavior," Econometrica 52, 10071028 (1984).

[6] Bernheim, B.D., B. Peleg and M. D. Whinston, "Coalition-proof Nash equilibria: I. Concepts," Journal of Economic Theory 42,1-12 (1987).

[7] Fudenberg, D. and D.K. Levine, The theory of learning in games, MIT Press (1998).

[8] Glicksberg, I.L., "A further generalization of the Kakutani fixed point theorem with application to Nash equilibrium points," Proceedings of the National Academy of Sciences 3, 170-174 (1952).

[9] Herings, P.J.J. and V. Vannetelbosch, "Refinements of rationalizability for normal-form games," International Journal of Game Theory 28, 53-68 (1999). 
[10] Herings, P.J.J. and V. Vannetelbosch, "The Equivalence of the Dekel-Fudenberg iterative procedure and weakly perfect rationalizability," Economic Theory 15(3), 677-687 (2000).

[11] Herings, P.J.J., A. Mauleon and V. Vannetelbosch, "Rationalizability for social environments," Games and Economic Behavior 49, 135-156 (2004).

[12] Hofbauer, J. and J.W. Weibull, "Evolutionary selection against dominated strategies," Journal of Economic Theory 71, 558-573 (1996).

[13] Hurkens, S., "Learning by forgetful players," Games and Economic Behavior 11, 304-329 (1995).

[14] Kalai, E. and D. Samet, "Persistent equilibria in strategic games," International Journal of Game Theory 14, 41-50 (1984).

[15] Kemeny, J. and J. Snell, Finite markov chains, Springer (1976).

[16] Kets, W. and M. Voorneveld, "Learning to be prepared," International Journal of Game Theory 37, 333-352 (2008).

[17] Pearce, D., "Rationalizable strategic behavior and the problem of perfection," Econometrica 52, 1029-1051 (1984).

[18] Voorneveld, M., "Preparation," Games and Economic Behavior 48, 403-414 (2004).

[19] Voorneveld, M., "Persistent retracts and preparation," Games and Economic Behavior 51, 228-232 (2005).

[20] Voorneveld, M., W. Kets and H. Norde, "An axiomatization of minimal curb sets," International Journal of Game Theory 33, 479-490 (2005).

[21] Weibull, J., Evolutionary game theory, MIT Press (1995).

[22] Young, H.P., Individual strategy and social structure, Princeton University Press (1998). 